\title{
LA CATASTROFE DEL 2008 EN LA PROVINCIA DE PALENA: REFLEXIONES SOBRE SUS PARTICULARIDADES E IMPACTO EN LA PATAGONIA OCCIDENTAL
}

\author{
FERNANDO MANDUJANO B.a \& JUAN C. RODRÍGUEZ T. ${ }^{\mathrm{b}}$
}

\section{RESUMEN}

La erupción volcánica del año 2008 sobre Chaitén, el poblado central de la provincia de Palena, fue el comienzo de una cadena de decisiones y conductas, algunas de las cuales resultan paradójicas. Ellas no fueron producto del error o la improvisación, sino que resultaron así a consecuencia de las especiales condiciones históricas, geográficas, económicas y políticas en que se produjo la catástrofe y que dejaron a sus protagonistas, tanto autoridades como víctimas, con márgenes de decisión muy limitados. A los enormes costos del desarraigo violento de un pueblo y la destrucción de su tejido social y sus rituales de vida cotidiana, se agrega un alto costo en una de las variables más críticas del desarrollo territorial de la Patagonia Occidental, que es justamente su dificultad secular para arraigar población y consolidar una red equilibrada de ciudades y poblados.

Un estudio de caso, con metodología mixta cuali-cuantitativa que combinó entrevistas a lugareños y autoridades en la localidad, con archivos de prensa y diversidad de artículos, documentos y estadísticas vinculadas al tema se utilizaron para hacer una descripción y avanzar a hacia una comprensión global de los procesos. ${ }^{1}$

PALABRAS CLAVE: Patagonia Occidental, provincia de Palena, catástrofe, policentrismo territorial.

\section{THE CATASTROPHE OF THE YEAR 2008 IN THE PROVINCE OF PALENA: SOME THOUGHTS ABOUT ITS CHARACTERISTICS AND IMPACT ON THE WESTERN PATAGONIA}

\section{ABSTRACT}

The volcanic eruption of 2008 on the town of Chaiten, in the middle of Palena Province, was the beginning of a series of decisions and behaviors, some of which result paradoxical. They were not the

a Facultad de Educación, Universidad de Playa Ancha, Avenida Playa Ancha 850, Valparaíso. fm@upla.cl

Facultad de Arquitectura Universidad de Valparaíso, Avenida el Parque 570, Valparaíso. juancarlosrodriguezt@yahoo.com

$1 \quad$ Este trabajo es producto del Proyecto FONDECYT 1161013: "De los ensamblajes locales, regionales y nacionales a los ensamblajes globales. Etnografía y política de las relaciones de sujeción local en el área de influencia de la Carretera Austral, la provincia de Palena y la región de Aysén". 
result of error or improvisation, but were so as a result of the special historical, geographic, economic and political conditions in which the disaster strock and left the protagonists, both authorities and victims, with very limited decision margins. Plus the enormous costs of violent uprooting of a town and the destruction of their social nets and rituals of everyday life, we must add a high cost in one of the most critical variables of territorial development at Western Patagonia, which is precisely its secular difficulty to ingrain population and consolidate a balanced network of cities and towns.

A case study with mixed quali-quantitative methodology that combined interviews with locals and authorities in the town, press archive and different articles, documents and statistics related to the subject, was used to make a description and advance towards a global understanding of the processes.

KEY WORD: Western Patagonia, province of Palena, catastrophe, territorial polycentrism.

\section{INTRODUCCIÓN}

...El hombre es el ser fronterizo que no tiene ninguna frontera, el cierre de su seren-casa por medio de la puerta significa ciertamente que separa una parcela de la unidad ininterrumpida del ser natural, pero asi como la delimitación informe se torna en una configuración, asi también su delimitación encuentra su sentido y su dignidad por vez primera en aquello que la movilidad de la puerta hace perceptible: en la posibilidad de salirse a cada instante de esta delimitación hacia la libertad...

George Simmel, 1998

Hasta mayo del 2008 el pueblo de Chaitén había avanzado en superar su aislamiento extremo de décadas, su crecimiento poblacional se acercaba a convertirlo según el INE en ciudad y por su ubicación geográfica central en la provincia de Palena, se configuraba como polo clave $y$ nodo funcional para el desarrollo territorial de la provincia y la Patagonia en general. A partir de esa fecha, naturaleza y actores sociales son lanzados, como en un guión de tragedia, en una secuencia de hechos y decisiones que en muchos aspectos significaron un gran retroceso y pérdida para la zona y sus habitantes.

La erupción del volcán Chaitén en la madrugada del viernes 2 de mayo del 2008, fue tan sorpresiva (Lara, 2009, p. 126) y violenta (Carn et al. 2009, p. 205), que las autoridades nacionales decretaron la evacuación inmediata de todos los habitantes del poblado del mismo nombre, ubicado a una decena de kilómetros.

Antes de la catástrofe, nadie en el pueblo sabía del peligro, no existía ningún conocimiento ancestral en la población sobre él, incluso los científicos sabían poco (OVDAS- SERNAGEOMIN, 2008 , p. 3). El volcán protagonista no fue el Michinmahuida como se afirmó al comienzo, sino uno vecino, más cercano, el denominado hasta ese día por los lugareños, Cerro Chaitén (Macaya, 2012, p. 5). Con el fin de la actividad, quedó establecido que el lugar de emplazamiento del pueblo es de importante riesgo, con una vulnerabilidad "exacerbada por la ocupación humana de un área de alto peligro volcánico" (Lara \& Calderón, 2015, p. 29). Todos los estudios que se hicieron a partir de esa fecha no modificaron ese veredicto fatal.

En menos de 4 días los 4.625 habitantes urbanos fueron evacuados (De la Barrera et al. 2011, p. 82). El día 6 de mayo, abandonan el lugar las últimas personas, el personal técnico y militar que condujo las operaciones. La rapidez y prioridades del desalojo hicieron que los evacuados dejaran prácticamente todos sus bienes, incluyendo sus mascotas, en las casas del pueblo abandonado.

Nunca antes en la historia de la Patagonia se había evacuado de manera tan urgente a una población tan numerosa radicada en un lugar. La salida no era optativa, las voces de expertos fueron claras: todas las personas debían ser evacuadas de inmediato.

Ese éxodo, en muchos sentidos, fue un final sin retorno para la mayoría. No más de una docena de lugareños regresaron durante ese año; al siguiente sumaban una treintena; en marzo del 2011 y decidida la reconstrucción del pueblo en 
el lugar original, sólo habían regresado unas 400 personas (Berezin, 2012, p. 17). A seis años, en 2014, según el Ministerio del Interior (2014, p. 7) se contaban 1.732 , pero además esa cifra considera una fracción importante de nuevos inmigrantes que se radicaron en el lugar, en un pueblo ahora divido por el río Blanco, entre los chaiteninos del norte y los de sur (1.180 y 552, respectivamente según el mismo informe), donde el sur es habitado por "familias que se encuentran en estos terrenos en calidad de "ocupas", en peores condiciones que las del Sector Norte, ya que la instalación de servicios es mínima" (op. cit.).

Muchas familias, la mayoría que vivía allí hasta esa fecha, no han vuelto; muchas que han regresado tuvieron sus propios cambios; hay muchas caras nuevas y desconocidas, especialmente en el Sector Sur, se señala. Gran parte de los que no regresaron fueron las personas de más edad, pues disminuyó fuertemente el rango etario de la población (Valenzuela, 2012, p. 18), es decir, muchos de aquellos que custodian la cultura local, la memoria y la historia oral en una comunidad, no han vuelto. Diversos indicadores señalan que cada vez es menos probable el regreso, especialmente cuando desaparece una tradición completa como la de los pescadores y algueros.

Reflexionando sobre cómo ha cambiado desde la catástrofe, un dirigente dice: "No existe algo colectivo en Chaitén, es muy difícil armarlo. Al llegar personas de otros lugares se perdió lo que teníamos de original acá. Un treinta por ciento de la gente no era de acá antes. Uno se encuentra a cada rato con personas que vienen de afuera, $y$ es difícil hacer comunidad con gente que uno no conoce, esas personas. Nosotros sabíamos quienes eran los vecinos, sabíamos los apellidos, sus problemas" (entrevista enero de 2014). Otro entrevistado, (citado en Arteaga \& Ugarte, 2015, p. 116) declara: "yo en lo particular siento nostalgia por el Chaitén del 2008, ese Chaitén que yo vi por última vez en la gruta antes de irme un día domingo".

La literatura socioantropológica indica que más allá del domicilio, el espacio urbano representa el lugar donde se desarrollan las prácticas sociales efectuadas en relación con los elementos materiales, la morfología y estructuración de las funciones (Delgado, 1999, pp. 13-24), el lugar donde se practica la vida colectiva, donde se asoman los indicios del barrio, las calles, los lugares para caminar y recrearse, el entorno de la ciudad; una topografía de la vida cotidiana que derivaba hacia el otro foco fundamental, el trabajo: "mientras el domicilio representaba un estar disponible para mí, un espacio vuelto permanentemente a mis requerimientos, con objetos a la mano para mi uso y goce personal, el trabajo representa el lugar de mi disponibilidad para los otros" (Giannini, 1999, p. 26)... "el domicilio es indicio y símbolo fuerte de la singularidad humana, así como la calle...símbolo de su universalidad, de su sociabilidad" (op. cit. p. 26). El estar para otros es lo que desaparece: el nombre propio, la identificación colectiva y el espacio público con las redes sociales y los rituales de la vida cotidiana que tenía el poblado hasta mayo del 2008.

La descripción de características claves de esa catástrofe, las complejas condiciones en que ocurre, fijando opciones de acción con mínimos grados de libertad, que se expresan en la secuencia de decisiones de autoridades y lugareños, especialmente sus distintas definiciones de la situación, y cómo esos discursos marcaron el desenlace de los acontecimientos hasta la situación actual, es el tema del presente artículo. La tesis sostenida es que la catástrofe es un retroceso en el desarrollo y ensamblaje territorial, se frustra la consolidación que presentaba Chaitén como posible polo urbano en una red de ciudades y poblados de carácter policéntrico y equilibrado para la Patagonia Occidental (ver mapa en Fig. 1), desechando una política territorial estratégica de mediano y largo plazo. También, se suma el abandono de un proyecto de ciudad ecológica modelo, la Nueva Chaitén; una idea fundacional frente a la realidad de las ciudades como Punta Arenas, Aysén, y especialmente Coyhaique, que evidencian un crecimiento no planificado y ambientalmente inarmónico. Esta última, incluso con altos niveles de contaminación, que la han llevado a ser la ciudad más contaminada de Chile y América según la OMS (WHO, 2016).

Con la idea de policentrismo, apuntamos a un proceso y a un objetivo. Como proceso, a las operaciones y lógica para establecer un orden virtuoso en términos territoriales, de conexión y redes, eliminando problemas de malformación como el aislamiento y desequilibrios asociados 
a la distribución de los bienes sociales, porque la lógica del ordenamiento territorial no puede estar supeditada al mercado o a una coyuntura, ya que genera vacío de la institucionalidad. Como objetivo, está asociado a la búsqueda -como fin- de la interconexión y complementariedad en términos de especialización económica, con impacto regional, que mejore el bienestar de las personas. De este modo, hablamos de equilibrio y sostenibilidad, desarrollo local, el uso y ocupación racional del territorio y gestión responsable de los recursos naturales, junto con la organización de la institucionalidad, la gestión, coordinación y la gobernabilidad de los territorios asociados para generación de un modelo de territorialidad más armónica e inclusiva.

La relevancia de esta lectura está en: 1) reconocer que nuestra institucionalidad actual y el centralismo de las decisiones han sido incapaces de pensar el territorio y subsistemas virtuosos y enlazados que permitan generar una integración policéntrica para la Patagonia, para consolidar asentamientos rurales que favorezcan el arraigo y la retentividad de la población; 2) hacer visibles las dificultades que un proceso tan complejo como una catástrofe natural plantea para la construcción de discursos consensuados, o, al menos compatibles y coherentes que permitan la coordinación y la gestión eficiente de esos procesos para restablecer la normalidad.

A pesar que la gestión de la catástrofe fue exitosa en salvar la vida de todas las personas y en que estas pudieran salir adelante con sus proyectos de vida en Chaitén mismo o en distintas localidades, la evaluación de los efectos de las decisiones presenta voces disonantes y contradictorias entre chaiteninos actuales, ex chaiteninos y analistas, los que hablan de segunda catástrofe: la social (Aguilera, 2009). En paralelo, y lo menos referido en la información secundaria, es que también dejó importantes costos y daños colaterales en el territorio.

Se presenta a continuación la metodología, vinculada con el marco conceptual del estudio dentro de un programa de investigación asociado a la territorialización de la Patagonia chilena. Luego, una caracterización de la Patagonia Occidental, de la provincia de Palena y de la comuna de Chaitén, para contextualizar los procesos estudiados y dejar en evidencia algunos aspectos críticos que van a ser afectados por la catástrofe, como la conectividad y aislamiento, la fragilidad de la ocupación territorial y su demografía, y la supervivencia de las culturas locales del vasto territorio.

\section{METODOLOGÍA}

Se usó una perspectiva cuali-cuantitativa con el método del estudio de caso tal como lo señala Yin (2003). Este se destaca por su utilidad -en vez de la encuesta, el experimento u otro- cuando se necesita hacer visibles procesos complejos. También propicia incorporar datos de situaciones imprevistas, tanto durante el trabajo de terreno, recolección de documentos, como en el análisis, incluyendo las interpretaciones y reinterpretaciones que surgen en un estudio de un fenómeno de esta densidad (Yin, 2003; Stake, 1978).

Desde los primeros datos que recogimos, la diversidad de las narraciones sugirió dejar el enfoque empirista, consistente en tratar esas narraciones como simples indicadores, y probar su tratamiento como herramientas de construcción social de la experiencia estudiada. La perspectiva de la hermenéutica social con su énfasis en el lenguaje fue la orientación adoptada para la realización del trabajo. Consideramos el Interaccionismo Simbólico con Blumer (1982) y Mead (1993), con su énfasis en cómo la interpretación diferenciada que hacen los actores modifica los significados que los mismos eventos tienen para ellos, según sus perspectivas e intereses y cómo esos significados se enlazan en conductas también diferentes. Durante los acontecimientos observamos que hay una construcción y reconstrucción de las relaciones y la estructura del sistema social de la comunidad (Stryker, 1987, p. 93), y la forma de asumir los roles y sus modificaciones por parte de los individuos, redefinen a su vez la estructura (Turner, 1988).

El trabajo de terreno es parte de un estudio mayor sobre los procesos de territorialización de la Patagonia. Se llevó a cabo en cuatro temporadas de campo: entre octubre y diciembre de 2012, marzo de 2013 y enero de 2014; en una macrozona considerada área de influencia de la Carretera Austral, abarcando recorridos por las provincias de Palena, Coyhaique, Aysén 
Fig. 1. Mapa Patagonia Occidental: Provincia de Palena, región de Aysén y Magallanes (conforme a cartografía IGM).

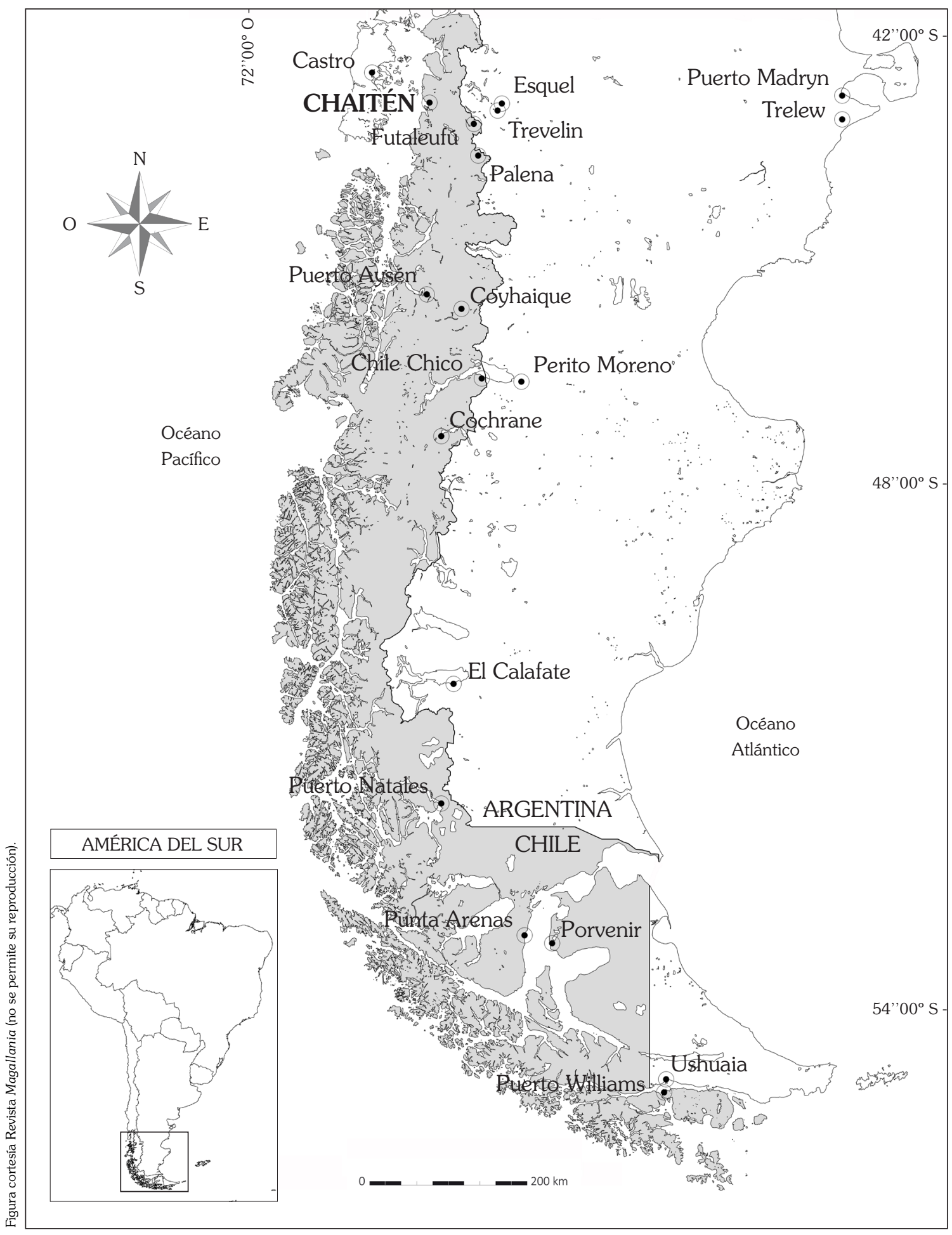


y General Carrera, concluidos en 2016. La importancia de lo ocurrido en Chaitén para la provincia de Palena y para toda la Patagonia, condujo a estudiar especialmente el caso. Para ello se elaboró un archivo de prensa, con el abundante material publicado desde el año 2008, a lo que se sumaron artículos e informes originados a partir de la catástrofe y su gestión, documentos y bases de datos públicos, transcripciones de las entrevistas realizadas en la localidad a informantes claves, la mayoría residentes "retornados", algunos con liderazgo local, provincial o nacional, sumadas a una decena de entrevistas casuales a lugareños, viajeros, transportistas, meseros, empleados de bencineras, comerciantes y un abundante material fotográfico.

El examen y la comparación de las narraciones permite mostrar complementación, cadenas de influencia, incongruencias, contradicciones y reformulaciones. Buscando formas de gestionar esa diversidad de narraciones, algunos autores han propuesto como herramienta metodológica alternativa la ciencia posnormal (Marshall \& Picou, 2008; Turnpenny et al. 2011), entendiéndola como una forma de acción donde las lentas pero duras certezas de la ciencia normal se conciliarían con las incertidumbres imperiosas de la acción política. Nuestra impresión es que las comunidades, no solo las locales, sino también las políticas y las académicas, construyen espacios de significados que son semánticos, densos y profundos, de difícil traducción y comunicación con otros, como sostuvo Geertz (2003). Y ello, por una parte, hace particularmente complejo el encuentro y la articulación entre la ciencia y la acción política en la gestión de los desastres; por otra, una combinación que se hace dramática en tales escenarios, desde los discursos que definen las situaciones y determinan sus consecuencias. Esa complejidad explica que este trabajo se proponga un primer avance en la formulación de reflexiones que permitan un estudio más integrado de los hechos.

2 En entrevistas a lugareños, se evidencia que la explicación no está asociada a una disminución de la natalidad, sino especialmente a un proceso de migración desde comunas y localidades rurales hacia los escasos y distanciados centros

\section{RESULTADOS}

\section{El Contexto:}

Chaitén y la Patagonia Occidental

En la Patagonia chilena sólo hay tres grandes centros urbanos: a unos $500 \mathrm{~km}$ lineales desde Puerto Montt al sur, nos encontramos con Coyhaique y Aysén (separadas entre sí por menos de 70), y luego a $840 \mathrm{~km}$, la ciudad de Punta Arenas (también en línea recta). Estas son las únicas ciudades con mercados significativos, servicios públicos y aeropuertos (Coyhaique y Punta Arenas) funcionando todo el año. Errores en las políticas de colonización, con grandes asignaciones de tierras a empresas que no concretaron poblados, que privilegiaron trabajadores solteros en vez de familias, y el Estado que no invirtió en infraestructura pública (caminos y conectividad), sumado a su difícil geografía, explican un poblamiento de baja densidad y más tardío que en el lado oriental de la Patagonia. Con menos de 1 habitante por $\mathrm{km}^{2}$, es la zona del país con menor densidad demográfica, acompañada por un bajo crecimiento de la población (INE 2012), pues sus tres regiones (Provincia de Palena en Los Lagos, Aysén y Magallanes) se encuentran dentro de las seis, del total de regiones de Chile, con más bajo crecimiento intercensal.

En la Tabla 1, se muestra el claro predominio en el territorio patagónico de comunas muy poco pobladas: del total de 24 , nueve tienen menos de un millar de habitantes.

Las cifras indican una tendencia inequívoca: mientras más pequeña es la población de la comuna, mayor es la probabilidad a perder población en el período intercensal ${ }^{2}$. Este desequilibrio en la ocupación territorial se muestra más complejo si consideramos que, al menos la mitad de la superficie de toda la Patagonia chilena está conformada por las comunas más críticamente aisladas del país (SUBDERE-PUC, 1999; SUBDERE, 2008, 2011).

La provincia de Palena, donde se localiza Chaitén, es la más septentrional de las ocho provincias que incluye la Patagonia Occidental.

urbanos de la Patagonia, porque "los niños terminan la escuela básica, y se van a la ciudad a continuar el liceo y no vuelven", señala un vecino de Bahía Murta en diciembre de 2012. 
Tabla 1. Comunas de la Patagonia Occidental * según tamaño de su población, Comunas que pierden población según tamaño, en el período intercensal. (INE, Censo 2012).

\begin{tabular}{|c|c|c|}
\hline Población & Comunas & Pierden Población \\
\hline Menos de 1.000 & 9 & 6 \\
\hline De 1.000 a menos de 5.000 & 9 & 4 \\
\hline De 5000 a menos de 10.000 & 2 & 0 \\
\hline De 10.000 a menos de 20.000 & 1 & 1 \\
\hline De 20.000 y más & 3 & 0 \\
\hline Total $=$ & 24 & 11 \\
\hline
\end{tabular}

Fuente: elaboración propia según datos INE. *Comunas incluidas en la Provincia de Palena y regiones de Aysén y Magallanes.

Está conformada por las comunas de Hualaihué, Chaitén, Futaleufú y Palena, y su poblamiento es tan reducido que, salvo Hornopirén, capital de Hualaihué, ninguno de sus agrupamientos habitacionales alcanza el estatus de ciudad conforme al criterio del Instituto Nacional de Estadísticas (INE), de 5.000 o más habitantes. También es la menos poblada de sus alrededores. Su densidad es de $0,9 \mathrm{hab} / \mathrm{Km}^{2}$ en comparación con Llanquihue, 24,4; Chiloé, 18,1; Aysén, 2,3; y Coyhaique, 4,6 (INE 2012).

La provincia es muy lluviosa y fría, con un paisaje de densos bosques, montañosa, atravesada por ríos correntosos y lagos encajonados, por lo que fue tardía y difícilmente colonizada desde territorio argentino y por incursiones estacionales desde Chiloé en busca de madera.

En la Tabla 2, se muestra la evolución de su población incorporando los censos de 1970 y 1982.
Aunque de norte a sur la ciudad de Hornopirén -en la comuna de Hualaihué- es la entrada geográfica a la zona, después de realizar un par de trasbordos en barcaza, el camino continuo termina allí. El inicio de la vía que se interna verdaderamente en la Patagonia, aparece al arribar por mar a Caleta Gonzalo, tramo de la inconclusa carretera Austral o ruta G7. Se trata de una de las obras civiles y de ingeniería más complejas, emprendidas por el Cuerpo Militar del Trabajo y llega hasta Villa O'Higgins, a unos 775 $\mathrm{km}$ al sur de Puerto Montt. Su tramo norte, hasta Chaitén, requiere la conexión por mar mediante transbordadores, lo que dificulta significativamente el ingreso a la Patagonia por territorio chileno (ver Fig. 2). Sin embargo, su trazado permitió convertir a Chaitén en puerto oceánico de entrada real para los que viajan por tierra a lo largo de esas regiones. De no usar la ruta marítima, el camino obligado para ir al Chile patagónico, interior y costero, es

Tabla 2. Población Provincia de Palena entre 1970 y 2012.

\begin{tabular}{lccccc}
\hline \multicolumn{1}{l}{ Comuna } & \multicolumn{5}{c}{ Censo } \\
\hline \multirow{2}{*}{ Chaitén } & 1970 & 1982 & 1992 & 2002 & 2012 \\
Futaleufú & 4.219 & 7.016 & 7.006 & 7.062 & 3.336 \\
Hualaihué & 2.398 & 1.809 & 1.737 & 1.822 & 2.299 \\
Palena & 5.526 & 6.302 & 7.872 & 8.210 & 8.702 \\
\hline \multicolumn{1}{c}{ Total } & 14.666 & 1.848 & 1.886 & 1.675 & 1.810 \\
\hline
\end{tabular}

Fuente: elaboración propia, datos INE.

3 Hualaihué es la única que tiene un crecimiento por su proximidad y su mayor accesibilidad a/desde Puerto Montt y por el boom de la salmonicultura y por la falta de conexión por tierra con el resto de la provincia de Palena, su presencia no tiene mayor impacto en el espacio provincial interior. 
por las carreteras argentinas.

La comuna de Chaitén es la más extensa $\left(8.471 \mathrm{~km}^{2}\right)$ de las cuatro de la provincia. $\mathrm{Su}$ ocupación fue difícil; data aproximadamente de 1921, cuando algunos navegantes de la Isla de Chiloé vinieron tras madera y trajeron los primeros animales. Descendientes de los pioneros cuentan que en los años 30 del siglo pasado, desplazarse entre la fronteriza Futaleufú -en la cordillera- y Chaitén -en la costa- demoraba unos 45 días. El tránsito con los animales se realizaba a través de la selva y había que "preparar picadas", es decir, abrir caminos a machete, lo que era peligroso por los derrumbes y las crecidas de ríos y lagos.

Hacia el interior, Futaleufú y Palena recién se transformaron por decretos supremos en municipalidad en 1971. Ambos pueblos están mayormente conectados e integrados con ciudades argentinas como Trevelin, Esquel y Bariloche, donde se atienden diversidad de necesidades alimentarias, recreacionales, de salud y educacionales, siendo estas dos últimas propiciadas por convenios del Estado chileno. La vinculación con el lado argentino es un rasgo dominante en lo que constituye una identidad patagónica compartida, un carácter binacional asociado al trabajo y comercio, con grandes contingentes de trabajadores radicados y temporales en el sur argentino; cuestiones parentales consanguíneas y políticas; también a redes que permanecen presentes en muchos aspectos de la cultura y la vida cotidiana. Hay radioemisoras que transmiten "para la Patagonia, chilena y argentina"; también, el valor del peso argentino constituye un indicador económico del día 4 .

Hasta comienzos de los años 60, Chaitén no tenía agua potable, luz eléctrica ni muelle, cuestión suficiente para llegar a conformar un mundo muy autónomo desde la perspectiva de las prácticas sociales, culturales y económicas, así como para resolver las contingencias en el día a día. Solo a partir del inicio de la construcción de la Carretera Austral, a fines de los años 70, experimenta los mayores cambios. Esto es descrito como "el gran avance" por la población, lo que consolidó a Chaitén como entrada y nodo articulador de la red vial y marítima de la zona. De este modo,

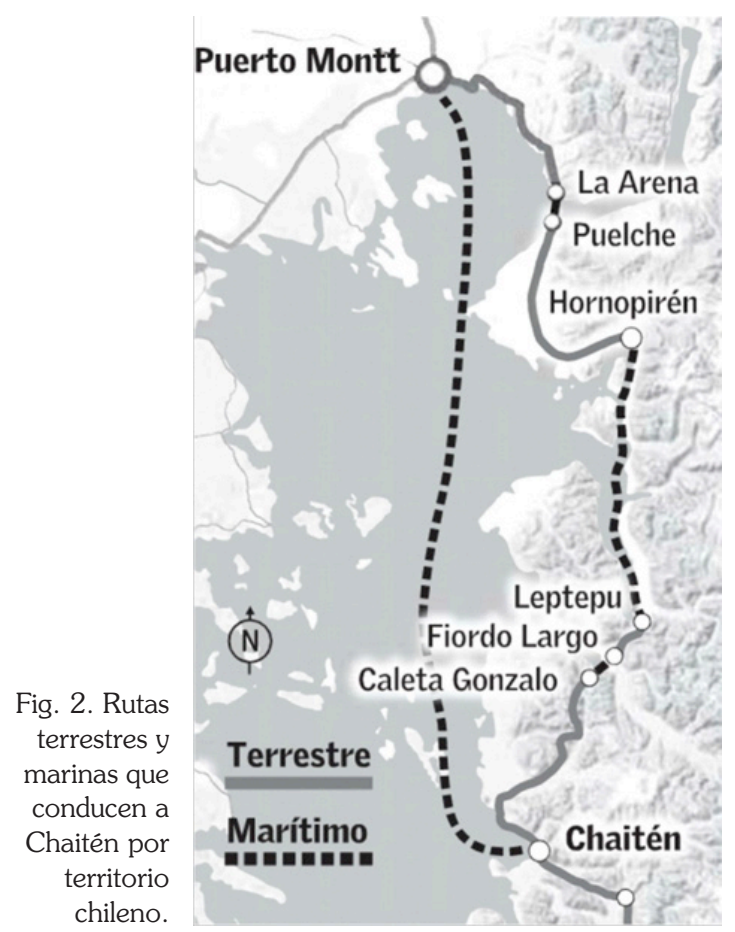

antes de la erupción volcánica, era el poblado más grande en más de $130 \mathrm{~km}$ a la redonda, que en los caminos de la Patagonia nunca son lineales, significan tiempos no controlables y mayores riesgos que en otras partes, ya que aún los cortes en las vías ripiadas son habituales por desbordes de ríos, derrumbes o caídas de árboles.

Así, en el año 2008 la comuna se aproximaba a los siete mil habitantes, de los cuales por lo menos unos cuatro mil vivían en el pueblo. La geografía de la provincia, sumada a lentos cambios, fortalecía la condición de localidad como confluencia natural de rutas de transporte, lugar de descanso y de abastecimiento, trasbordo, distribución de productos y servicios. Ubicada en un sector central, donde la Ruta Austral confluye con el puertoembarcadero y el mejor aeródromo provincial, con más días utilizable (reconstruido unos kilómetros al norte), instalaron las condiciones para que llegara a ser el centro urbano con más habitantes y centro de servicios públicos y comercio para todas las localidades y actividades de la Provincia.

El informe de la Subsecretaria de Desarrollo Regional (SUBDERE, 2008, p. 25), justo antes

4 Esto muestra que para gran parte de la zona austral el poblamiento no ha sido norte/sur, como se cree etnocéntricamente desde el Chile central, porque hay un claro predominio de carácter bidireccional oeste/este. 
de la catástrofe, saca a Chaitén del listado de las comunas de aislamiento crítico donde había aparecido en el informe equivalente del año 1999 (SUBDERE-PUC), bajando del Quartíl más crítico, el Q1 al Q3, aislamiento medio. En el informe de la misma Subsecretaría del año 2011 (SUBDERE, 2011, p. 71), es decir, con la situación post erupción, devuelve al pueblo a la lista de las localidades más aisladas.

\section{La Erupción del Volcán Chaitén:}

Relación de los acontecimientos y las decisiones

El primer informe técnico del Servicio Nacional de Geología y Minería (SERNAGEOMIN, 3 mayo 2008) decreta Alerta Roja, la que se define por parte de la Oficina Nacional de Emergencia (ONEMI) "cuando el evento crece en extensión y severidad, requiriendo la movilización de todos los recursos necesarios y disponibles, para la atención y control del evento destructivo. Una Alerta Roja se puede establecer de inmediato con la amplitud y cobertura necesarias, sin que medie previamente un Alerta Amarilla, según las características de la situación". Esta alerta significó evacuación inmediata ${ }^{5}$, y se va a extender por dos años, hasta junio del 2010.

Aunque no existen actas públicas de las deliberaciones que tomaron las autoridades durante la gestión de la catástrofe, se puede hipotetizar que las decisiones que se estudiaron debieron ser básicamente las de la Fig. 3.

En junio de 2010 SERNAGEOMIN baja su evaluación de riesgo a Alerta Amarilla, pero indica: "Dada la gran dimensión del domo, continúa latente el peligro de colapsos, eventuales explosiones y generación de flujos de bloques y ceniza, los cuales podrían afectar los valles adyacentes al volcán Chaitén" (Neira, 2010). El caso del cambio de alerta, no significa liberar al poblado de su vulnerabilidad y la viabilidad de mantener ahí su emplazamiento, lo que está avalado por la autoridad de acuerdo a informes científicos. De este modo, constituye el punto cero para las decisiones que tomaron las autoridades en lo sucesivo.

Distribuir a los refugiados en varias ciudades resultó, al parecer, bastante mejor decisión. Se evacuó unas 8.000 personas de Chaitén y otras localidades como Futaleufú, hacia otras regiones sin perder una vida (IFRC, 2008). Casi la mitad de las 1.820 familias evacuadas los primeros días fueron ubicadas en refugios habilitados en colegios de Puerto Montt (900), un 22\% en localidades de Chiloé, y un $12 \%$ en localidades muy pequeñas alcanzables por tierra, como La Junta, Palena, Villa Santa Lucía (Programa de Apoyo a Chaitén, 2008).

En junio de 2008 regresan masivamente chaiteninos a recoger enseres y constatan la gravedad de los daños y pérdidas, ahí surgen críticas

Fig. 3. Árbol de decisiones básicas tomadas en la gestión de la catástrofe.

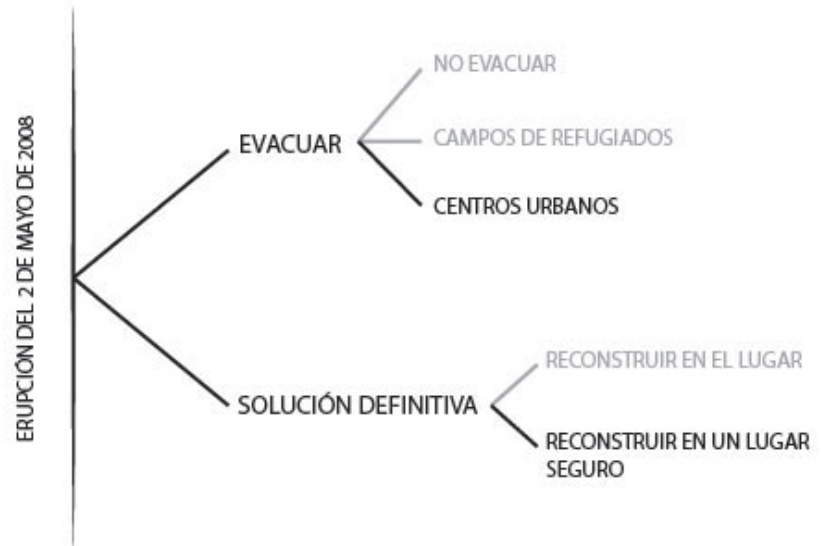

5 Incluyó evidentes dificultades para el transporte terrestre, por la visibilidad afectada por la lluvia de cenizas y acumulación en la ruta. Podemos suponer que la disponibilidad de materiales, mano de obra adecuada para construir un campamento, las condiciones de transporte del territorio, sumada a las dificultades y precariedad de servicios y comodidades de un campamento ad hoc, fue menos recomendable que la opción que efectivamente se adoptó: localizar a las familias en espacios urbanos, dotados de infraestructura como agua potable, electricidad, comercio, centro de salud, escuelas, telefonía y servicios del Estado. 
a las autoridades, culpándolas por los daños de la catástrofe: "por no permitir la recuperación de bienes antes de la crecida del río" (Observatorio de Ciudades UC, 2009, p. 89). Luego, en el análisis de decisiones se observa la segunda catástrofe: la desarticulación familiar y social.

En una catástrofe "normal", o más frecuente, como terremotos, incendios $e$ inundaciones, las escuelas y estadios techados son refugios habituales, pero por unos días o semanas. La gran diferencia en este caso fue que además de no poder regresar a sus casas y su pueblo, la duración de la actividad volcánica es de curso y extensión impredecibles. De este modo, si en la fase final de la gestión de un desastre se trata de la normalización de la vida (Herrmann, 2007, p. 13), aquí aparece otra condicionante paradojal para la toma de decisiones, sin grados de libertad: la normalización de la vida se tenía que hacer lejos del lugar de origen. En sentido estricto: volver a la normalidad sin volver a ella.

En la siguiente serie de decisiones no se tuvieron casi alternativas al frente: la condición de refugiados no podía extenderse indefinidamente hasta que se pudiese regresar al pueblo, o regresar al pueblo reconstruido en otra localidad alternativa. Si la primera opción no era viable a la luz de los veredictos técnicos que declaran la inhabitabilidad, la segunda tomaría de todas formas muchos meses, al menos demasiado tiempo para restablecer la normalidad en la vida de las familias. Entonces, esas personas tenían derecho a tratar de reconstruir sus vidas dónde se encontraban o dónde quisieran, lo más pronto posible.

\section{La normalidad paradójica:}

la comunidad fragmentada

La pérdida del hogar, el desplazamiento y refugio obligado en localidades lejanas, sin duda fueron hechos dramáticos. El dolor y el estrés sufridos por la comunidad, las familias y las personas, están entre los más extremos (Berroeta et al. 2015, p. 54) y quizá, sólo superables por las pérdidas de vidas que podrían haber ocurrido. Probablemente esa diferencia en la memoria de muchas víctimas, frente el dolor real vivido, haga aparecer como irreal el riesgo que obligó a evacuar, incluso percibir la propia evacuación como innecesaria y cómo el inicio de la catástrofe.

El gobierno central buscó apoyo experto, con alrededor de 80 profesionales dedicados a la asistencia directa de la población afectada en las localidades de refugio (Aguilera, 2009). Sumó el asesoramiento científico de su gestión, consultando y contratando servicios en las diversas disciplinas vinculadas al problema, incluyendo a instituciones chilenas (SERNAGEOMIN, OVDAS, universidades) y extranjeras (Arup, VHP-USGS ${ }^{6}$, NASA (2008)). En suma, se dictaminó que los pobladores requerían más que el simple refugio o albergue inicial: necesitaban un programa de apoyo continuo en un proceso de meses o años de asistencia y relocalización de las familias.

La dura experiencia de ser "sacados de sus casas y de su pueblo" y sin posibilidad de retorno, condujo a las autoridades a un esfuerzo sin precedentes de mitigación, que significó entregar a los damnificados un conjunto de ayudas:

a) Asignación de bono para arriendo, en cualquier lugar escogido

b) Dinero para los adultos, en una comuna con ingresos promedios más bajos que los de la región y el país

c) Asistencia médica y sicológica

d) Becas y cupos para estudio de los hijos

e) Cursos de capacitación y apoyo técnico para la reconversión laboral

f) Oferta de compra de sus viviendas destruidas, a valores previos a la catástrofe (Ley $\mathrm{N}^{\circ}$ 20.385, del 7 de octubre de 2009).

g) El Ministerio de Vivienda y Urbanismo (MINVU) creó un alto subsidio especial para la vivienda, a fin de facilitar el acceso a una nueva, ascendente a 670 UF.

Sin embargo, los tiempos políticos, académicos y familiares comienzan a ser tempranamente asincrónicos e incompatibles. Así, la adaptación en otras localidades más grandes y lejanas, mejor dotadas de servicios, con más opciones de comercio y acceso a bienes, estudio 
para los hijos, de trabajo, diversión, autoexpresión, conocimiento de personas, que las que ofrecía el pequeño pueblo y la provincia antes de la catástrofe, mermaron la urgencia del retorno, especialmente entre aquellos que encontraron refugio en centros urbanos mejor dotados (Mandujano et al. 2015; Rodríguez et al. 2016)

En el interludio de las decisiones del Estado, los consultores y los refugiados, las personas que tuvieron mayor facilidad para regresar a mirar con sus propios ojos lo acontecido, recuperar o eventualmente reinstalarse, un grupo se escinde y se transforma en un grupo contestatario: los llamados o autoproclamados "rebeldes". Un conjunto de vecinos con posiciones sociales ventajosas y posesiones difíciles de replicar en otros lugares, que insisten -muy tempranamenteen la reconstrucción de Chaitén y que van a resultar claves para decidir el destino final del pueblo. Con ello, se rompe el soporte comunitario; se polarizan los vecinos entre los "vendidos al Estado" y los "auténticos chaitenínos", quienes insistieron que el pueblo debía ser reconstruido en el mismo emplazamiento.

Éstos, desde los primeros días de la evacuación resistieron y discreparon de todos los diagnósticos oficiales que dieron por destruida la localidad, por lo que el gobierno se vio obligado a solicitar autorización ante los tribunales de justicia para obligarlos a abandonarla. Prescindiendo de la evidencia científica, concertados en la apreciación empírica y subjetiva que el Sector Norte no estaba tan afectado, ellos asumieron que era "habitable", incluso después de las peores salidas del río?.

Uno de los líderes avecindado desde el año 1988, narra así su experiencia en entrevista realizada en enero de 2014: "Primero regresé en lancha, después cuando ganamos el recurso de protección que autorizaba a quedarse en el poblado, pude regresar en el mes de julio y me vine por Argentina en julio del 2008, y luego se instaló mi vecina al lado que vendía pan, ella vendía pan; porque esto seguía siendo puerto, y los barcos en espera necesitaban que alguien les vendiera pan. Y ella me decía: "Oye, no te vayas de aquí, quédate acá, yo te doy almuerzo, si quieres venir a tomar once, si quieres venir a la cena, pero no te vayas de acá. Entonces uno sentía que si uno de los cinco, cuatro, seis o diez que habíamos [sic] se iba [sic], es como que mucha gente se iba. Entonces me costaba mucho salir de acá".

En la misma entrevista, tratando de explicar por qué se quedó y el origen de su protagonismo entre los rebeldes nos agrega: "Yo me había quedado, porque la radio estaba funcionando... era el único medio local. Es más, las otras dos personas se iban a la radio a escuchar las trasmisiones. Además, salíamos por la Internet. Era importante, porque nos escuchaban de todas partes para saber qué ocurría en la ciudad".

A pocos meses de la evacuación total, regresan. Y pese a las prohibiciones, según sus palabras, "hacen funcionar el pueblo": instalan bombas de agua, generadores eléctricos e importan clandestinamente combustible desde las ciudades argentinas de Esquel y Trevelin. Protagonizan diversas protestas, donde algunos enarbolan banderas argentinas y aparecen por primera vez en la zona las bombas lacrimógenas de la policía antidisturbios. Por cierto, un episodio que será indeleble y épico que reforzará la posición de Chaitén en Chaitén.

La imposición de este criterio de fuerza, no técnico, por parte de quienes tenían inversiones mayores a escala local, termina siendo funcional a algo que también realmente constituía una dificultad política para las autoridades: invertir en una zona de riesgo. Según los expertos, era imposible para el gobierno aceptar o promover la reconstrucción en un lugar de tanto riesgo.

El argumento utilizado para la recuperación de Chaitén por este grupo de "rebeldes", se encuentra siempre con un refuerzo de acusación a la autoridad por negligencia. Pese a la gran inversión de fondos públicos descrita, para proteger a la población y facilitar su relocalización, con medidas asistidas por especialistas competentes, el discurso que sobrevive es más bien crítico del rol del Estado. Ugarte y Salgado (2014, p. 157) lo señalan de la siguiente forma: "Se percibe al Estado como el principal responsable del desastre, en la medida en que sus acciones tienen como consecuencia la dispersión territorial de las familias y comunidad en general, la prohibición de retornar al territorio y

7 Un diagnóstico casi textual, va a usar dos años después la administración del Presidente Piñera, cuando anuncia la reconstrucción en su lugar original. 
la incertidumbre constante respecto a su futuro"; y, agregan: "[el Estado] es percibido, por sobre la presencia del volcán, como la principal amenaza en el territorio".

En los pobladores sobrevive en la actualidad un discurso que en muchos aspectos se relaciona muy poco con los de otros actores y en otros momentos. El veredicto experto de vulcanólogos y geólogos que declaran inhabitable el poblado no es recordado y es omitido por una dirigente vecinal, que declara: "... el desastre, por llamarlo así de alguna forma, comienza cuando la autoridad se empeña en... en sacar a la gente" (Ugarte \& Salgado, op cit. p. 151).

Ugarte y Salgado (op. cit. p. 152) recogen narraciones de lugareños durante 2012 y en base a ellas afirman: "la erupción del volcán pasa a segundo plano frente al desborde del río Blanco, evento definido como desastroso en la medida en que se encuentra asociado a una acción inoportuna por parte del Estado; cuyos funcionarios, desconociendo las dinámicas propias del territorio, no logran tomar las decisiones correctas en el momento apropiado; por ejemplo, los habitantes solicitaron el drenaje del río, pues intuían que podría desbordarse; sin embargo, el Estado no acoge esta demanda. De este modo, el momento en que comienza la intervención del Estado sobre el territorio de Chaitén es señalado como el inicio del desastre; esto debido a que la inundación de la ciudad obliga la evacuación y dispersión de la población chaitenina".

Evidentemente estos relatos no concuerdan con lo descrito al inicio de este artículo: la evacuación se hizo en el mismo día, dentro de las 48 horas de iniciada la gran erupción. El primer desborde del río se produjo más de una semana después, y los pobladores refugiados en Puerto Montt y otras localidades, vieron en los noticieros las imágenes de las casas arrasadas, con el pueblo vacío. Ninguna autoridad habría enviado máquinas ni operadores a despejar el río justo al lado de un volcán en actividad, que según los expertos podía borrar el pueblo en cualquier momento y a la entrada del crudo invierno de la Patagonia. Salvo estos recuerdos nuevos, no existen evidencias de una petición de despeje o dragado del río en esos primeros días y tampoco las prioridades de la emergencia que se vivía eran propicias para que se le prestara atención, si es que la hubo.

Este proceso de re-narración que va modificando los recuerdos, es un área que amerita otro estudio, pues altera la definición de las situaciones y por lo tanto, sus consecuencias. Chabris y Simons (2011, p. 101) denominan "ilusión de la memoria" a la diferencia entre "cuán precisos son nuestros recuerdos, y cuán precisos creemos que los son". Estas distorsiones, por ejemplo, pueden modificar lo que queda instalado como "lecciones aprendidas" de la gestión de las catástrofes. Remarcando el protagonismo de los "rebeldes", no sólo -como dice Ginzburg (2010, p. 20)- una afirmación verdadera, y una inventada no parecen diferentes, y las verdades, igual que los conceptos, son definidas por su uso. Usos que según Schütz (1974, p. 97), dependen de los intereses de los sujetos y grupos, pues como esos intereses son cambiantes, especialmente en periodos de crisis, las reinterpretaciones que dan de los hechos los pobladores son también cambiantes.

La consecuencia del discurso de este grupo de vecinos rebeldes, que está más allá de la primera y segunda tragedia, es que ellos producen la reafirmación de la posibilidad del asentamiento original del pueblo. Y, además, definen una nueva estratificación y distribución espacial: ellos quedan con posiciones ventajosas, los funcionarios públicos y municipales con sus asignaciones de zona, algunos antiguos que se apropiaron de casas compradas por el Fisco, y los ocupas del sector sur.

También podemos suponer que esa desigualdad interna de la comunidad está asociada a narraciones distintas y que los costos y beneficios se distribuyeron de manera desigual por diferencias sociales, políticas y económicas internas, un dato clave en un paradigma de la vulnerabilidad y de análisis en condiciones críticas como las descritas. Unos, estaban más adentro, y otros más afuera de Chaitén. Siguiendo a Alicia Lindón (Lindón, 2007, p. 42), "en un lugar concreto y en un tiempo igualmente demarcado... están presentes otros lugares que actúan como constituyentes de ese lugar. Esos otros lugares traen consigo otros momentos o fragmentos temporales, otras prácticas y actores diferentes aunque también pueden ser semejantes a las que se están realizando en ese escenario". Son imaginarios distintos que articulan lo material y lo ideacional; con prácticas, lugares, escenarios, sen- 
tidos e intencionalidades que marcan la tridimensionalidad de todo lugar: lo material, las prácticas y acciones, y los significados.

\section{El Proyecto de Relocalización en una ciudad modelo, la única localidad abandonada}

En el mes de octubre, a casi seis meses del suceso, el gobierno contrata con la Pontificia Universidad Católica de Chile un estudio para reconstruir o relocalizar Chaitén. El informe se recibe durante el primer semestre del 2009; en él se confirma la inhabitabilidad del pueblo, se propone la nueva ubicación y se presenta el primer proyecto de ciudad modelo ecológica. La relocalización propuesta indica Santa Bárbara, caserío ubicado 10 kilómetros al norte. El resultado se expresa en un proyecto de ciudad modelo, en sintonía con la identidad de futuro de la Patagonia y acreditada como ciudad ecológica (Green Globe Brand Certified \& Benchmarked, PUC, op. cit. p. 127) y de conformidad a cómo también lo querían los chaiteninos dispersos, quienes habían sido convocados a participar de focus group y entrevistas, según el informe.

Sin embargo, a pocos meses, en el mismo año, el Intendente Regional anuncia que se descarta la propuesta PUC, por el costo de los predios privados que contempla, y el MINVU con sus propios equipos profesionales, elabora un nuevo proyecto para la Nueva Chaitén, asentado en la misma localidad de Santa Bárbara, sobre suelos fiscales.

Llegado el año 2010, se producen dos hechos que cambian radicalmente el escenario de los acontecimientos: se produce el gran terremoto y tsunami de febrero, y un cambio de gobierno en marzo donde asume la administración del Presidente Piñera. Aun así, a los pocos días de asumido el nuevo gobierno, el MINVU entrega El Plan Maestro Ciudad Chaitén (División de Desarrollo UrbanoMINVU, 2010). El informe propone "una ciudad con una fuerte identidad paisajistica y cultural, que incorpore criterios de sustentabilidad ambiental, social y económica, que garantice una alta calidad de vida para sus habitantes, y que se consolide como la puerta de acceso a la Carretera Austral".

En mayo, el nuevo gobierno confirma la construcción en Santa Bárbara (Diario La Nación,
27 de mayo 2010). Pero, transcurrido casi un año, en abril de 2011 (Radio Cooperativa, 9 de abril 2011), cambia de decisión y anuncia la reconstrucción de Chaitén en el sector norte del mismo pueblo, en lo que denomina "Plan Solución Chaitén". Con ello, ocurren al menos 4 cuestiones: 1) se abandonan las propuestas de la PUC y del MINVU, 2) se impone la propuesta de los "rebeldes", es decir, la reconstrucción en el mismo emplazamiento, 3) se define la escala demográfica en función de las viviendas disponibles y recuperables, y 4) se refuerza la idea de una ausencia de política demográfica-territorial para la Patagonia.

Pese al gran esfuerzo realizado, primero por las universidades y luego por los profesionales de la División de Desarrollo Urbano del MINVU, el proyecto de relocalización en Santa Bárbara no prosperó. Todo indica que no logró algún nivel de arraigo ni despertó confianzas en las expectativas de los habitantes. Ni en los que vivieron, al menos en los primeros meses el extrañamiento o refugio con la esperanza de volver, ni en aquellos que se negaron a abandonar el pueblo. El costo del proyecto, en momentos de urgentes demandas para la reconstrucción por los daños del terremoto de febrero, debió resultar una cifra exorbitante para el Estado.

La fragmentación de la comunidad en grupos repartidos en muchas partes no sólo propició a la larga el debilitamiento de la cohesión, las organizaciones locales y el apego con Chaitén, asociable al bajo nivel de retorno observable, sino también esa dispersión debe haber dificultado crecientemente la validez de diversas encuestas y dinámicas para estudiar las expectativas de estas personas respecto del proyecto Nueva Chaitén. Simultáneamente, esa fragmentación geográfica dificultó el desarrollo de un discurso compartido para la solución que se adoptaría finalmente.

Las facilidades para reinsertarse en las nuevas localidades propiciadas por el Programa de Apoyo a las Familias, se ve acentuada no sólo como hemos señalado más arriba por la compra por parte del Fisco de la vivienda abandonada en la evacuación, sino adicionalmente con el subsidio preferencial para la compra de otra. También consolidan la dispersión, otras iniciativas como las becas y cupos para estudio de los hijos, programas 
de capacitación, reconversión laboral y terapia. Es decir, un conjunto de facilidades que con el paso de los meses hicieron cada vez más viable y atractivo recomenzar la vida en los lugares de acogida.

En cambio, frente a estas facilidades para adaptarse a sus nuevos lugares de vida, las que también deben ser leídas como oportunidades, la ciudad de Chaitén se empezó a percibir de manera más intensa como precaria y su condición de habitable fue siempre "una completa incertidumbre" (González, 2011, p. 119), ya que nunca estuvo claro si se podía volver a la misma, ni cuándo, así como tampoco hubo señales de que verdaderamente se estuviera reconstruyendo la Nueva Chaitén en Santa Bárbara. En muchos de nuestros registros de campo se repite la siguiente sentencia: "Todo se veía bello, pero todos sabíamos que no se podía construir en medio del mallín" (entrevista en marzo de 2013); o, "si nunca nos pescaron para nada, menos les íbamos a creer que iban a construir una ciudad de lujo" (entrevista en marzo del 2013).

Este último testimonio, refuerza la idea que Chaitén con todos los que se fueron y llegaron, es un lugar nuevo. Con menos comunidad local, menos fortalezas en términos de cohesión social para discutir presupuestos participativos, consultas sobre desarrollo local y asambleas o juntas vecinales que permitan alcanzar resultados solidarios con impactos endógenos. Si los rebeldes en su momento capturaron o neutralizaron el ofrecimiento del Estado de la ciudad modelo, e impusieron no moverse de la localidad, con esa experiencia épica y organizativa quedaron con un poder muy importante para cooptar las decisiones municipales o provinciales.

Sin embargo, a pesar de la construcción del Aeródromo Nuevo Chaitén y nuevos tramos de la Carretera Austral, todo se comienza a reducir paulatinamente a una situación de problemas locales de la pobreza o programas y proyectos focalizados en esa dirección y no de desarrollo territorial policéntrico. Es evidente que existe implícitamente en el curso de hechos y decisiones que, se produce un retraso de la política de reequilibrio espacial que permitiría reconfigurar positivamente la relación centro periferia que caracteriza al país, especialmente en la Patagonia. La evaluación final, es que la intervención en Chaitén terminó siendo una política focalizada de emergencia, la que permitió el control de unos pocos actores, pero sin desarrollo endógeno porque hubo un menoscabo demográfico.

Ello, obliga a decir que: nuevamente la Patagonia pierde en este nodo vial y marítimo. Como sostiene Serge Watcher (2000), el desafío del ordenamiento territorial es hacer compatibles los territorios institucionales de orden político administrativos; los funcionales de corte económico y financiero; $y$, los de carácter patrimonialista, que son los vividos en el cotidiano.

\section{CONCLUSIONES}

La presencia de la modificabilidad del recuerdo en la historia local es una primera observación. Estas revisiones a lo narrado, con modificaciones reiteradas, lo observa en la fenomenología Schütz, en su análisis del conocimiento del nativo, lo hace la historiografía, destacándolo como un problema central, en Ginzburg (2010) y Marc Bloch (2001) y desde la neurociencia, autores como los citados Chabris y Simon (2011). Aquí aparecen en pocos meses, un par de años, las re narraciones. Sólo que ésta vez lo hace una comunidad de lugareños, alfabetizados, que interactúa con lectoescritura y en redes sociales.

También se destaca cómo una minoría autónoma y bien organizada, pasa a tener un protagonismo determinante: "los rebeldes". La forma en que ejercen su rol es destacable, durante el primer año no fueron más de 15 personas, pero su control de la única radio del lugar, y su capacidad para generar soluciones de resiliencia y protagonismo frente a los medios externos fue central. Al año siguiente, el 2009, siguieron siendo una minoría de no más de 30 personas, como hemos señalado. Sin embargo, la efectividad de sus protestas con las banderas negras y argentinas, los posicionaron como los interlocutores privilegiados en la representación del pueblo. La reconstrucción del mismo en su emplazamiento original, a expensas del riesgo, tuvo en sus accionar un factor decisivo. El poder y efectividad de esta minoría, probablemente tuvo algún efecto de modelaje en las organizaciones de protestas de Aysén y Magallanes que siguieron al poco tiempo.

En este estudio de caso, por lejos se debe destacar la catástrofe demográfica, y lo que podemos denominar su impacto en la habitabilidad y la territorialización de esta zona de la Patagonia. 
Queda de manifiesto, pese al esfuerzo del Estado y recursos comprometidos, que la política territorial es subsumida a cuestiones de orden compensatoria y reactiva, pero escasamente proactiva. A más de seis años del suceso, el pueblo se encuentra aun dañado y minimizado; menos de un tercio de su población ha regresado. La pérdida de población significa no sólo una reducción del estímulo "a la formación de la red de relaciones a partir de los intercambios aleatorios que suscita la ciudad" (Rémy \& Voyé, 2006, p. 12), ya que también es un empobrecimiento del potencial de desarrollo y calidad de vida del hinterland rural y el sistema de pueblos y localidades con los cuales intercambian servicios. El ideal de la red equilibrada y policéntrica de asentamientos urbanos para una territorialización armoniosa de la Patagonia, acusa la profundización de esta fractura en su ensamblaje.

La sinergia de la interacción humana es muy sensible al número de participantes y a sus características. Los cambios de la población actual de Chaitén, en relación a la existente hasta mayo del 2008, están asociados a alteraciones profundas en la vivencia de la vida cotidiana, las que aparecen en algunos recuerdos y percepciones de lugareños, y cuyo seguimiento puede ayudar a indagar el proceso de re narración de la historia y la identidad local.

El aislamiento descrito que sufren las localidades de la provincia de Palena es también un costo para las generaciones actuales y próximas. La pérdida demográfica sufrida por Chaitén tiene un efecto en cadena sistémico: en términos cuantitativos, es un mercado más pequeño, afectando las posiblidades de economías de escala, que afecta precios $y$ diversidad, que refuerza las escasez alimentaria de las zonas vecinas (las localidades de food desert). Ello es especialmente complejo en un país que deja al mercado como principal o único asignador de recursos, aún con el peso que tiene la política de subsidios y asignaciones de zona para funcionarios públicos de zonas aisladas. Y, en términos cualitativos produce un impacto no sólo por el tipo de pobladores que ser perdió, tema sobre al cual se dispone poca información; como ocurre en todas las migraciones humanas, éstas no son aleatorias, porque el tipo de habitantes que quedan define también el perfil ocupacional y productivo de la localidad.

Asimismo, el caso Chaitén tiene impacto en la visibilidad y presencia de la Patagonia (peso en decisiones políticas, posibilidades de emprendimiento local). Esto también sugiere una cierta fragilidad del apego, ya visible en las estadísticas censales, y que aparece acá cuando observamos los datos del retorno: ¿por qué han regresado tan pocos? ¿cuántos más abandonarían localidades de la Patagonia si tuviesen la posibilidad? ¿existe - como dicen Azócar et al. (2010, p. 102) "un agotamiento y cansancio latente"? El que la mayor parte de las familias hayan decidido no regresar cuando se les facilitó la opción de radicarse en las ciudades de refugio, dice algo respecto de lo dura que puede resultar la vida en zonas aisladas.

Si en la Patagonia la demografía es frágil, con un crecimiento reducido que se concentra en dos o tres escenarios urbanos, mientras el aislamiento crítico es un rasgo de la mayor parte de su territorio, son justamente las comunas más aisladas las que más sufren ese despoblamiento. La catástrofe urbana y demográfica de Chaitén no deja de ser, en alguna forma, un menoscabo a la habitabilidad de la provincia y de todo el territorio: es un fortalecimiento a la condición de frontera interior, de zona apartada de Chile y de falta de visión en términos de ordenamiento territorial del Estado.

El largo e incierto proceso de recuperación y las definiciones estratégicas sobre Chaitén como puerta de entrada de la Patagonia, que parece detenido o abandonado, impone al Estado una reflexión y una tarea: cómo resolver o disminuir la brecha en el sistema de oportunidades en el país interior e integrar la variable disparidad territorial a las políticas públicas en términos de gestión y programas. En este sentido, la condición de aislamiento, dificultades de desplazamiento y carencia de servicios especializados y de calidad, constituyen un criterio inexcusable de diferenciación territorial; $y$, por lo mismo, debiese ser susceptible la localidad, como el conjunto de las comunas del interior de la provincia de Palena, de discriminación positiva

La vida en Chaitén se reanuda, pero ahora con una siniestra amenaza que lo acompañará silenciosa, como un apocalipsis propio que en cualquier momento puede activarse, el cuarto volcán más peligroso del territorio chileno (SERNAGEOMIN, 2015). 


\section{BIBLIOGRAFÍA}

Aguilera, R. (2009). Chaitén: El Lado B de la Tragedia. Revista Nos. Febrero 2009. Recuperado 10 enero 2010: http://www.revistanos.cl/2009/02/chaitenel-lado-b-de-la-tragedia/

Arteaga A. C., \& Ugarte, A. M. (2015) Identidades En Emergencia: La Otra Cara de la Reconstrucción. El Caso de Chaitén. Magallania, 43(3),107-213

Azócar, G., Aguayo, M., Henríquez, C., Vega, C., \& Sanhueza, R. (2010). Patrones de crecimiento urbano en la Patagonia chilena: el caso de la ciudad de Coyhaique. Revista de Geografía Norte Grande, $46,85-104$

Berezin, A. (2012). Chaitén: una Historia en el Lugar. Tesis para optar al título profesional de sociólogo. Universidad de Chile, Santiago.

Berroeta, E., Ramoneda, A., Rodríguez, V., di Masso, A., \& Vidal, T. (2015). Apego de Lugar, Identidad de Lugar, Sentido de Comunidad y Participación Cívica en Personas Desplazadas de la Ciudad de Chaitén. Magallania, 43(3), 51-63.

Bloch, M. (2001). Apología para la Historia o el Oficio de Historiador. México: F C E.

Blumer, H. (1982): Interaccionismo Simbólico. Barcelona: Editorial Hora.

Carn, S., Pallister, J., Lara, L., Ewert, J., Watt, S., Prata, A. J., Thomas, R. J., \& Villarosa, G. (2009): The Unexpected Awakening of Chaitén Volcano, Chile, EOS Transactions American Geophysical Union. The Newspaper of the Earth and Space Sciences, 90, 24, 16. 205-206.

Chabris, C., \& Simons, D. (2011). El Gorila Invisible. $Y$ otras maneras en que nuestra intuición nos engaña. Madrid: Siglo XXI Editores.

De la Barrera, F., Reyes-Paecke, S. \& Meza, L. (2011). Análisis del paisaje para la evaluación ecológica rápida de alternativas de relocalización de una ciudad devastada. Revista Chilena de Historia Natural, 84, 181-194.

Delgado, M. (1999). El animal público. Barcelona: Anagrama.

DiarioLa Nación 27 mayo 2010. PiñeraConfirmaReubicación de Chaitén en Santa Bárbara. Recuperado el 12 de noviembre de 2012: http://www.lanacion.cl/ pinera-confirma-reubicacion-de-chaiten-en-santabarbara/noticias/2010-05-27/180052.html

División de Desarrollo Urbano-MINVU (2010): Plan Maestro Ciudad Chaitén. Informe Final. Ministerio de
Vivienda y Urbanismo. Recuperado 5 octubre 2012: http://www.plataformaurbana.cl/wp-content/ uploads/2010/04/1270556064planchaiteninform efinal.pdf

Geertz, C. (2003). La interpretación de las culturas. Barcelona: Gedisa.

Giannini, H. (1999). La Reflexión cotidiana, hacia una arqueología de la experiencia, Santiago de Chile: Editorial Universitaria

Ginzburg, C. (2010). El Hilo y Las Huellas. Lo verdadero, lo falso, lo ficticio. México: Fondo de Cultura Económica.

González, M. (2011). Estudio del impacto territorial ambiental generado por la erupción del volcán Chaitén. Memoria para optar al título de geógrafa, Escuela de Geografía, FAU, Universidad de Chile.

Herrmann, J. (2007). Disaster Response Planning \& Preparedness: Phases of Disaster. NY: New York Disaster Interfaith Services (NYDIS).

IFRC(14 may 2008): Chile: VolcanicEruptionDREFOperation No. MDRCL003. International Federation of Red Cross And Red Crescent Societies. Recuperado 15 enero 2013: http://www.redhum.org/archivos/ pdf/ID2232RedhumCLDREFOperationNo. MDRCL003VolcanicEruption.pdf

INE (2012). Resultados Censo de Población y Vivienda 2012. Santiago de Chile. Recuperado 10 abril 2013: http://www.ine.cl

Lara, L. (2009). The 2008 Eruption of Chaitén volcano, Chile: A preliminary report. Geological Note. Andean Geology, 36(1), 125-129.

Lara, L., \& Calderón, R. (2015). Peligro y riesgo específico asociado al volcán Chaitén: Perspectiva geológica de la vulnerabilidad en el entorno de un volcán activo". Magallania, 43(3), 27-35

Lindón, A. (2007). Los imaginarios urbanos y el constructivismo geográfico: los hologramas espaciales. EURE 33(99), 31-46.

Macaya P. (2012) Volcán Chaitén: consecuencias y discusiones. Ensayo en teoría y política ambiental. Santiago: Universidad de Heidelberg-UCH-PUC.

Mandujano, F., Rodríguez, J., Reyes, S., \& Medina, P. (2015). La erupción del volcán Chaitén: voyerismo, desconfianza, academia y Estado. Consecuencias urbanas y sociales en la comunidad. Universum, 30(2), 153-177.

Marshall, B., \& Picou, J. (2008). Postnormal Science, Precautionary Principle, and Worst Cases: The Challenge of Twenty-First Century Catastrophes. 
Sociological Inquiry, 78, 2. 230-247

Mead, G. (1993). Espíritu, Persona y Sociedad. Desde el punto de vista del conductismo social. México: Editorial Paidós.

Ministerio del Interior (2014). Diagnóstico Estado de la Reconstrucción. Erupción Chaitén 2 de mayo de 2008. Gobierno de Chile.

NASA (2008). Earth Observatory: Chaitén Volcano Erupts. Recuperado 5 noviembre de 2012: http:// earthobservatory.nasa.gov/NaturalHazards/view. php?id=19887

Neira, S. (2010, 2 de junio). Informe de Sernageomín: Volcán Chaitén entra en Fase de Alerta Amarilla. El Mercurio. Recuperado de: http://www.emol.com/ noticias/nacional/2010/06/02/416613/informede-sernageomin-volcan-chaiten-entra-en-fase-dealerta-amarilla.html

Observatorio de Ciudades UC. (2009). Informe Final Etapa 3 - Junio 2009). Recuperado 4 octubre de 2012: http://www.goreloslagos.cl/ resources/descargas/acercadegore/docgestion/ chaiten/3erinformeversionfinal.pdf

OVDAS-SERNAGEOMIN (2008). Erupción del Volcán Chaitén Primer Informe Técnico, 3 de Mayo de 2008. Gobierno de Chile. Consultado en mayo de 2016, en: http://ign.gob.ar/descargas/cnuggi/ chaiten.pdf

Radio Cooperativa 9 abril 2011: Piñera dio a conocer "Plan Solución Chaitén" y oficializó refundación de la ciudad. 9 abril 2011. Recuperado 7 noviembre 2012 en: http://www.cooperativa.cl/noticias/ pais/region-de-los-lagos/volcan-chaiten/pineradio-a-conocer-plan-solucion-chaiten-y-oficializorefundacion-de-la-ciudad/2011-04-09/223055. html

Rémy, J., \& Voyé, L. (2006) La ciudad ¿hacia una nueva definición?. Zaragoza: Bassarai Ediciones.

Rodríguez, J.,Reyes, S., \& Mandujano, F. (2016). El proyecto nueva Chaitén: La asincronía entre Estado, academia y comunidad. Revista AUS 19. 73-79.

SERNAGEOMIN (2008). Erupción del volcán Chaitén. Primer informe técnico, 03 de mayo de 2008 OVDAS-SERNAGEOMIN. Consultado en: http:// www.ign.gob.ar/descargas/cnuggi/chaiten.pdf

SERNAGEOMIN (2015). Ranking de los 90 volcanes activos de Chile. Consultado en: http://www. sernageomin.cl/archivos/Ranking-de-Volcanes.pdf

Simmel, G. (1998) Puente y Puerta. Recuperado el 23 de Diciembre de 2013, en http://laasociacion. files.wordpress.com/2013/05/simmel_1_libropuente_y_puerta-1.pdf

SUBDERE-PUC (1999). Diagnóstico y propuestas para la integración de territorios aislados. Santiago: LOM Ediciones.

SUBDERE (2008). Actualización Estudio Diagnóstico y Propuesta para Territorios Aislados. Santiago: Ministerio del Interior.

SUBDERE (2011). Estudio Identificación de Territorios Aislados 2011. Santiago: Ministerio del Interior y Seguridad Pública.

Schütz, A (1974). Estudios sobre teoría social. Buenos Aires: Amorrortu Editores.

Stake, R. (1978). The Case Study Method in Social Inquiry. Educational Researcher, 7, 2. 5-8.

Stryker, S. (1987). The Vitalization of Symbolic Interactionism. Social Psychology Quarterly, 50, 1. 83-94.

Turner, J. H. (1988). A theory of Social interaction. Stanford, CA, Stanford University Press.

Turnpenny, J., Jones, M., \& Lorenzoni, I. (2011). Where Now for Post-Normal Science?: A Critical Review of its Development, Definitions, and Uses. Science, Technology \& Human Values, 36. 287-306.

Ugarte, A., \& M. Salgado (2014). Sujetos en emergencia: acciones colectivas de resistencia y enfrentamiento del riesgo ante desastres; el caso de Chaitén, Chile. Revista INVI, 80, 29, 143-168.

Valenzuela, A. M. (2012). Análisis socioeconómico para la localidad de Chaitén después de la erupción del volcán homónimo y posterior aluvión del río Blanco. Memoria para optar al título de Geógrafo. U. Austral de Chile. Valdivia.

Watcher, S. (2000). Repenser le territoire. Un Dictionnaire critique, DATAR/ EDITIONS, Paris: de l' Aube.

WHO (2016). WHO Global Urban Ambient Air Pollution Database. Consultado el 15 de mayo 2016, en: http://www.who.int/phe/health_topics/outdoorair/ databases/cities/en/

Yin, R. (2003). Case Study Research. Design and Methods. USA: SAGE Publications. 
